\title{
Research on Height Control Algorithm for Hydro-Pneumatic Suspension System Based on Model Predictive Control
}

\author{
LIU Shang-hong ${ }^{a}$, LIU Chang ${ }^{b}$, ZHAO Yu-zhuang ${ }^{c}$ \\ School of Mechanical Engineering, Beijing Institute of Technology, Beijing 100081, China \\ alg5220063@163.com, ' lcworkemail@163.com, cbitzyz@163.com
}

Keywords: hydro-pneumatic suspension; height control; model predictive control

\begin{abstract}
Aiming to improve the precision of vehicle height control for hydro-pneumatic suspension system, having deeply considered the nonlinear factors existing in the lifting and lowering process, we derived the equations of hydraulic pressure in the cylinder and air pressure gradient in the accumulator. Based on these mathematical descriptions, a nonlinear quarter car hydro-pneumatic suspension model was established. The nonlinear model was globally linearized and discretized, and a model predictive control was designed in the linear domain. The simulation results show that the designed controller is able to solve the overshoot problem existing in the lifting and lowering process of hydro-pneumatic suspension and improves vehicle height control precision and quality obviously.
\end{abstract}

\section{Introduction}

Hydro-pneumatic suspension, due to its elastic property of nonlinear variable stiffness and oil flow rate control according to the driving situations, can effectively improves vehicle ride comfort and handling stability [1]. The oil in the cylinder and the air in the accumulator are in parallel. Due to the compressibility of air and the hysteresis of proportional solenoid valve, overshoot phenomenon will be caused in the lifting and lowering process.

In this paper, height control in the lifting and lowering process was studied. For the nonlinear factors of charging and discharging, the oil pressure gradient equation in the cylinder and the air pressure and volume gradient equation in the accumulator were established. Then model predictive controller is designed by linearizing and discretizing the vehicle height adjustment nonlinear model. By comparing the response of vehicle height under different controllers, it is fully verified that the model predictive control algorithm can effectively solve the overshoot problem and its control effect is better than other control strategies'.

\section{Dynamic model}

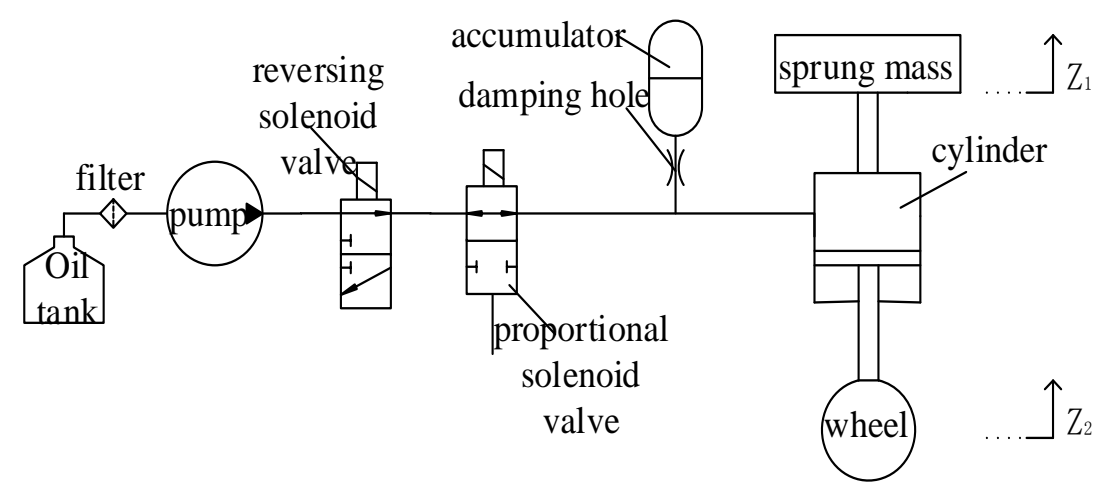

Fig. 1 structure of hydro-pneumatic suspension system

Dynamic model of hydro-pneumatic suspension. Structure of hydro-pneumatic suspension system is shown in Fig. 1. Vehicle body is supported by a hydro-pneumatic suspension actuator. By controlling the direction of electromagnetic valve and the opening of proportional solenoid valve to adjust oil flow rate in the charging and discharging process, it can realize vehicle height control [2]. 
In equilibrium state, by doing force analysis of hydro-pneumatic suspension system, the kinetic equation is available as follows.

$$
\begin{gathered}
\ddot{z}_{1}=\frac{1}{M_{1}}\left[P_{\mathrm{h}} A_{1}-M_{1} g-C\left(\dot{z}_{1}-\dot{z}_{2}\right)\right] \\
\ddot{z}_{2}=\frac{1}{M_{2}}\left[-P_{\mathrm{h}} A_{1}-M_{2} g+C\left(\dot{z}_{1}-\dot{z}_{2}\right)-K_{\mathrm{t}} z_{2}\right]
\end{gathered}
$$

Where $M_{1}, M_{2}$-sprung mass and unsprung mass, $Z_{1}, Z_{2}$-displacement of sprung mass and unsprung mass, $A_{1}$-effective area of cylinder piston, $P_{\mathrm{h}}$-oil pressure in the cylinder, $C$-equivalent damping coefficient.

By modeling air compressing process in the accumulator, we can obtain from air adiabatic equation that

$$
P_{0} V_{0}^{\eta}=P_{\mathrm{A}} V_{\mathrm{A}}^{\eta}
$$

Take derivative of time in the Eq. 3.

$$
\dot{V}_{\mathrm{A}}=-\frac{V_{\mathrm{A}}}{P_{\mathrm{A}} \eta} \dot{P}_{\mathrm{A}}
$$

Where $\eta$-heat ratio is constant, and we usually take $1.3, P_{0}, V_{0}$-air pressure and volume in equilibrium state, $P_{\mathrm{a}}, V_{\mathrm{a}}$-instantaneous air pressure and volume.

Furthermore, the volume of oil flowing into (or out of) the accumulator is equal to the reducing (or increasing) volume of air.

$$
\dot{V}_{\mathrm{A}}=-\operatorname{sign}\left(P_{\mathrm{h}}-P_{\mathrm{A}}\right) C_{\mathrm{d}} A_{3} \sqrt{\frac{2\left|P_{\mathrm{h}}-P_{\mathrm{A}}\right|}{\rho}}
$$

Air pressure gradient in the accumulator can be obtained from Eq. 4 and Eq. 5.

$$
\dot{P}_{\mathrm{A}}=\operatorname{sign}\left(P_{\mathrm{h}}-P_{\mathrm{A}}\right) C_{\mathrm{d}} A_{3} \sqrt{\frac{2\left|P_{\mathrm{h}}-P_{\mathrm{A}}\right|}{\rho}} \frac{P_{\mathrm{A}} \eta}{V_{\mathrm{A}}}
$$

In the process of charging and discharging, there exists a relative movement of cylinder and piston. Oil volume is obtained as follows.

$$
V_{\mathrm{h}}=V_{\mathrm{h} 0}+A_{1}\left(z_{1}-z_{2}\right)
$$

Set $Q$-total flow rate through proportional solenoid valve, $Q_{1}$-flow rate flowing into the cylinder,

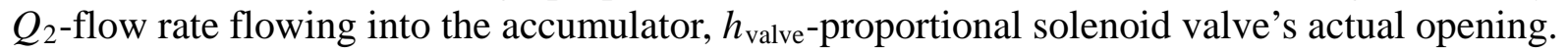

Considering the compressibility of oil, pressure increment of oil is equal to the product of relative value of volume and volume compressibility factor [3].

$$
\begin{gathered}
\dot{P}_{\mathrm{h}}=\beta \frac{Q_{1}-A_{1}\left(\dot{z}_{1}-\dot{z}_{2}\right)}{V_{\mathrm{h}}} \\
Q_{1}=Q-Q_{2} \\
A_{v}=A_{2} h_{\text {valve }} \\
Q_{2}=\operatorname{sign}\left(P_{\mathrm{h}}-P_{\mathrm{A}}\right) C_{\mathrm{d}} A_{3} \sqrt{\frac{2\left|P_{\mathrm{h}}-P_{\mathrm{A}}\right|}{\rho}}
\end{gathered}
$$

In the vehicle height adjustment process,

$$
Q=C_{\mathrm{d}} A \sqrt{\frac{2\left(P_{\mathrm{u}}-P_{\mathrm{d}}\right)}{\rho}}
$$

Dynamic model of system. Based on the hydro-pneumatic suspension system model and the proportional solenoid valve model, we established the dynamic model. It can be expressed by the following state equations [4].

$$
\begin{aligned}
& \dot{\boldsymbol{x}}(t)=f(\boldsymbol{x}(t), \boldsymbol{u}(t)) \\
& \boldsymbol{y}(t)=g(\boldsymbol{x}(t), \boldsymbol{u}(t))
\end{aligned}
$$


Where the input $\boldsymbol{u}=\left[h_{\text {valve }}\right]$, the state vector $\boldsymbol{x}=\left[z_{1}, \dot{z}_{1}, z_{2}, \dot{z}_{2}, P_{\mathrm{h}}, P_{\mathrm{A}}, V_{\mathrm{A}}\right]^{T}$.

According to the equations (1), (2), (6), (8),

$$
\begin{gathered}
f_{1}=\dot{x}_{1}=x_{2} \\
f_{2}=\dot{x}_{2}=\frac{1}{M_{1}}\left[x_{5} A_{1}-C\left(x_{2}-x_{4}\right)\right]-g \\
f_{3}=\dot{x}_{3}=x_{4} \\
f_{4}=\dot{x}_{4}=\frac{1}{M_{2}}\left[-x_{5} A_{1}+C\left(x_{2}-x_{4}\right)-K_{\mathrm{t}} x_{3}\right]-g \\
f_{5}=\dot{x}_{5}=\beta \frac{Q_{1}-A_{1}\left(x_{2}-x_{4}\right)}{V_{\mathrm{h}}} \\
f_{6}=\dot{x}_{6}=\operatorname{sign}\left(x_{5}-x_{6}\right) C_{\mathrm{d}} A_{3} \sqrt{\frac{2\left|x_{5}-x_{6}\right|}{\rho} \frac{x_{6}}{x_{7}}} \eta \\
f_{7}=\dot{x}_{7}=-\operatorname{sign}\left(x_{5}-x_{6}\right) C_{\mathrm{d}} A_{3} \sqrt{\frac{2\left|x_{5}-x_{6}\right|}{\rho}}
\end{gathered}
$$

Besides, the Eq. 10 can be expressed as

$$
A_{v}=A_{2} x_{8}
$$

\section{Control Algorithm}

Since the system model is nonlinear, the model needs to be linearized and discretized before the design of model predictive controller. The main feature of model predictive control is explicitly dealing with constraints of the controlled object on the basis of known model. Through online rolling optimization which take the characteristics of the system into account, output the optimal controlled variable to the controlled object so that the deviation of controlled variable and target value becomes as small as possible, and it meets expected target ultimately [5].

The main constraint conditions of hydro-pneumatic suspension system is the controller's output value. It means that the input value of proportional electromagnetic valve varies from 0 to 1 .

$$
0 \leq h \leq 1
$$

Set predictive width as $N_{\mathrm{p}}$, control width as $N_{\mathrm{c}}$, and $N_{\mathrm{p}} \geq N_{\mathrm{c}}$ [6]. The system quadratic rolling optimization target of the reference trajectory matrix $\boldsymbol{Y}_{\text {ref }}(k)$ and model prediction output matrix $\boldsymbol{Y}(k)$ can be written as

$$
\begin{gathered}
J_{\mathrm{k}}=\left\|\boldsymbol{Y}(k)-\boldsymbol{Y}_{\text {ref }}(k)\right\|_{\mathbf{Q}}^{2}+\|\Delta \boldsymbol{U}(k)\|_{\mathbf{R}}{ }^{2} \\
\boldsymbol{Y}(k)=\left[\begin{array}{c}
y(k+1 \mid k) \\
\vdots \\
y\left(k+N_{\mathrm{P}} \mid k\right)
\end{array}\right], \boldsymbol{Y}_{\text {ref }}(k)=\left[\begin{array}{c}
y_{\text {ref }}(k+1 \mid k) \\
\vdots \\
y_{\text {ref }}\left(k+N_{\mathrm{P}} \mid k\right)
\end{array}\right], \Delta \boldsymbol{U}(k)=\left[\begin{array}{c}
\Delta u(k \mid k) \\
\vdots \\
\Delta u\left(k+N_{\mathrm{C}}-1 \mid k\right)
\end{array}\right]
\end{gathered}
$$

Where $Q, R$-weighted matrix of output variables and control variables, $\Delta U(k)$-increment matrix of predictive control. The weighted matrix $R$ is to suppress the control increment from too severe, in order to prevent the system going beyond limits [7].

Prediction equation of y can be written as $\boldsymbol{y}(k+j \mid k)=\boldsymbol{C}\left(t_{i}\right) \boldsymbol{x}(k+j \mid k), j=1, \ldots, N_{\mathrm{p}}$. Then we can get

$$
\boldsymbol{y}(k+j \mid k)=\boldsymbol{\Phi} \boldsymbol{x}(k)+\boldsymbol{\Gamma} \mathbf{u}(k-1)+\boldsymbol{G}_{\boldsymbol{y}}\left[\begin{array}{c}
\Delta u(k \mid k) \\
\vdots \\
\Delta u\left(k+N_{\mathrm{C}}-1 \mid k\right)
\end{array}\right]
$$




$$
\boldsymbol{\Phi}=\boldsymbol{C}\left(t_{i}\right)\left[\begin{array}{l}
\boldsymbol{A}\left(t_{i}\right) \\
\vdots \\
\boldsymbol{A}^{N_{\mathrm{C}}\left(t_{i}\right)} \\
\boldsymbol{A}^{N_{\mathrm{C}}+1}\left(t_{i}\right) \\
\vdots \\
\boldsymbol{A}^{N_{\mathrm{P}}}\left(t_{i}\right)
\end{array}\right], \boldsymbol{\Gamma}=\boldsymbol{C}\left(t_{i}\right)\left[\begin{array}{l}
\boldsymbol{B}\left(t_{i}\right) \\
\vdots \\
\sum_{i=0}^{N_{\mathrm{C}}-1} \boldsymbol{A}^{i}\left(t_{i}\right) \boldsymbol{B}\left(t_{i}\right) \\
\sum_{i=0}^{N_{\mathrm{C}}} \boldsymbol{A}^{i}\left(t_{i}\right) \boldsymbol{B}\left(t_{i}\right) \\
\vdots \\
\sum_{i=0}^{N_{\mathrm{P}}-1} \boldsymbol{A}^{i}\left(t_{i}\right) \boldsymbol{B}\left(t_{i}\right)
\end{array}\right], \boldsymbol{G}_{\boldsymbol{y}}=\boldsymbol{C}\left(t_{i}\right)\left[\begin{array}{lll}
\boldsymbol{B}\left(t_{i}\right) & \cdots \\
\boldsymbol{A}\left(t_{i}\right) \boldsymbol{B}\left(t_{i}\right)+\boldsymbol{B}\left(t_{i}\right) & \cdots & 0 \\
\vdots & \ddots & \vdots \\
\sum_{i=0}^{N_{\mathrm{C}}-1} \boldsymbol{A}^{i}\left(t_{i}\right) \boldsymbol{B}\left(t_{i}\right) & \cdots & \boldsymbol{B}\left(t_{i}\right) \\
\sum_{i=0}^{N_{\mathrm{C}}} \boldsymbol{A}^{i}\left(t_{i}\right) \boldsymbol{B}\left(t_{i}\right) & \cdots & \boldsymbol{A}\left(t_{i}\right) \boldsymbol{B}\left(t_{i}\right)+\boldsymbol{B}\left(t_{i}\right) \\
\vdots & \vdots & \vdots \\
\sum_{i=0}^{N_{\mathrm{P}}-1} \boldsymbol{A}^{i}\left(t_{i}\right) \boldsymbol{B}\left(t_{i}\right) & \cdots & \sum_{i=0}^{N_{\mathrm{P}}-N_{\mathrm{C}}} \boldsymbol{A}^{i}\left(t_{i}\right) \boldsymbol{B}\left(t_{i}\right)
\end{array}\right]
$$

Define $E(k)=\boldsymbol{Y}_{\text {ref }}(k)-\boldsymbol{\Phi} \boldsymbol{x}(k)-\boldsymbol{\Gamma} \mathbf{u}(k-1)$.

Then Eq. 24 can be represented as the following constrained optimization problem.

$$
\min _{\Delta \boldsymbol{U}(k)} J_{\mathrm{k}}=\frac{1}{2} \Delta \boldsymbol{U}^{T} \boldsymbol{H} \Delta \boldsymbol{U}(k)+\boldsymbol{f}^{T} \Delta \boldsymbol{U}(k)+\text { const, } \quad \boldsymbol{H}=2\left(\boldsymbol{G}_{y}{ }^{T} \boldsymbol{Q} \boldsymbol{G}_{y}+\boldsymbol{R}\right), \boldsymbol{f}=-2 \boldsymbol{G}_{y}{ }^{T} \boldsymbol{Q} E(k)
$$

As shown in Eq. 23, it is the constraint condition. It is a typical quadratic programming problem and the optimal control vector increment matrix $\Delta U(k)$ is obtained [8].

According to the rolling optimization principle of predictive control, the controller solves the constrained optimization problems online at each sampling time, but it just outputs the first of the optimal control vector to the controlled object model. And during the next sampling time it solves the constrained optimization problem again so as to realize rolling optimization. The structure of MPC system is shown in Fig. 2.

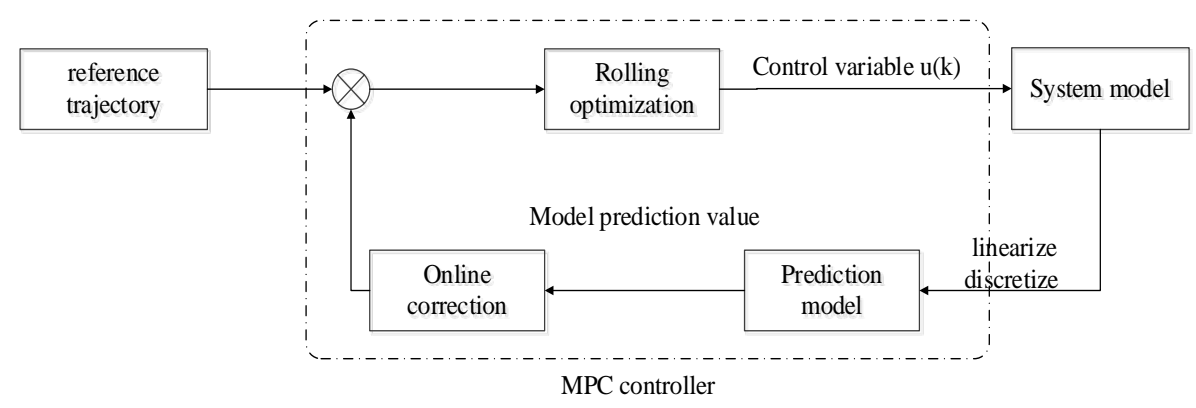

Fig. 2 structure of MPC system

\section{Results and discussions}

Based on the model predictive controller, we studied the effect of control algorithm via MATLAB/Simulink simulation. The main parameters are shown in table 1.

\begin{tabular}{|c|c|c|}
\hline Symbol & Value & Units \\
\hline$m_{1}$ & 1000 & [kg] \\
\hline$m_{2}$ & 100 & [kg] \\
\hline$K_{t}$ & 1000000 & {$[\mathrm{~N} / \mathrm{m}]$} \\
\hline$A_{1}$ & 0.0028 & {$\left[\mathrm{~m}^{2}\right]$} \\
\hline$P_{\text {oil }}$ & 6000000 & {$[\mathrm{~Pa}]$} \\
\hline$V_{0}$ & 0.001 & {$\left[\mathrm{~m}^{3}\right]$} \\
\hline$P_{0}$ & 4852457.46 & {$[\mathrm{~Pa}]$} \\
\hline$V_{h 0}$ & $5.6549 \mathrm{e}-004$ & {$\left[\mathrm{~m}^{3}\right]$} \\
\hline
\end{tabular}

Table 1. Main parameters for height control system 


$\begin{array}{lcc}A_{2} & 3.1416 \mathrm{e}-006 & {\left[\mathrm{~m}^{2}\right]} \\ A_{3} & 7.0686 \mathrm{e}-006 & {\left[\mathrm{~m}^{2}\right]} \\ \beta & 6.9 \times 10^{8} & {\left[\mathrm{~N} / \mathrm{m}^{2}\right]} \\ C & 5000 & {[\mathrm{~N} / \mathrm{m}]} \\ \left.K_{x}\right] & 1 & {[\mathrm{rad} / \mathrm{s}]} \\ \omega & 15 & \\ \xi & 0.7 & {[\mathrm{~Pa}]} \\ P_{\mathrm{b}} & 3.4660 \mathrm{e}+006 & \end{array}$

The response of vehicle height during the lifting process is shown in Fig. 3. The MPC controller's sampling period $T$ is $0.001 \mathrm{~s}$.

The Fig. 3 shows that there exists a big overshoot in the lifting process under switch control for the compressibility of air in the accumulator. PID control 1 can effectively solve the overshoot problem, but its adjustment response speed is slow. In order to increase the response speed of PID control 1, PID control 2 is developed, but it will cause a certain overshoot. In contrast, the model predictive control can effectively improves the precision and response speed of vehicle height control.

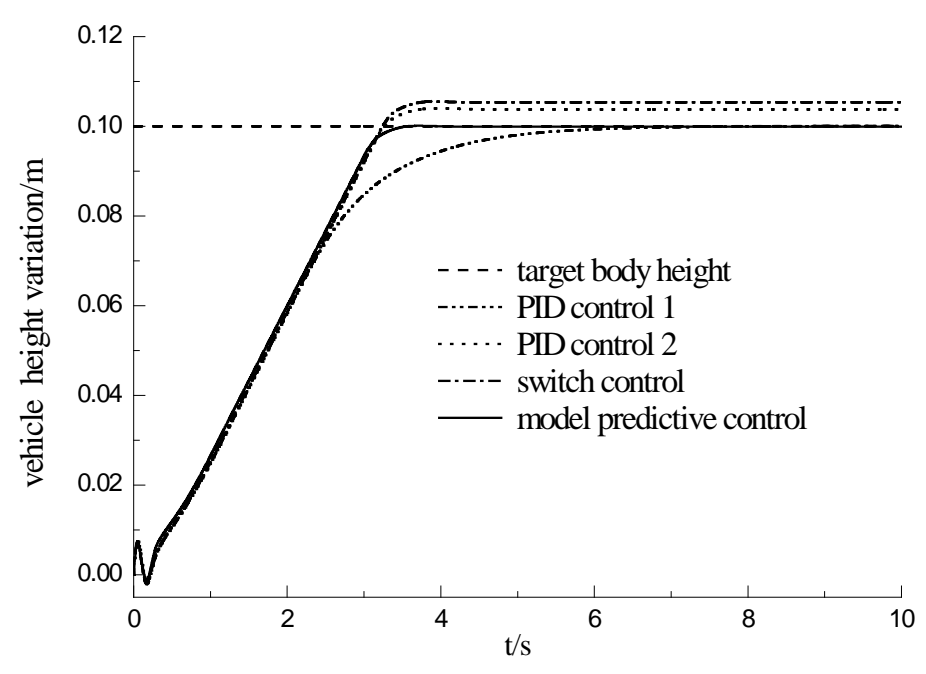

Fig. 3 vehicle height in the lifting process

\section{Conclusion}

According to the dynamic model of hydro-pneumatic suspension system, the height adjustment model was established. Through online rolling optimization, vehicle height can be forecasted. By outputting the control signal of optimal opening of proportional solenoid valve, we control the charging and discharging processes to achieve expected target height. The simulation results show that model predictive controller can solve the overshoot problem quickly and steadily compared with the switch control and PID control, improving the quality and precision of vehicle height control.

\section{Fund Project}

Project supported by the National Natural Science Foundation of China (Grant No. 51375046 and Grant No. 51205021).

\section{References}

[1] Zhao Yuzhuang. Research on Nonlinearity and Damping Control Strategy of Hydro-Pneumatic Suspension [D]. Beijing: Beijing Institute of Technology, 2011. (in Chinese) 
[2] Chen Zhilin, et al. Nonlinear Modeling, Simulation and Test Verification of Hydro-pneumatic Active Suspension [J]. Automotive Engineering, 2000, 22(3): 209-213. (in Chinese)

[3] Han Zhanzhong, Wang Guoyu. Fundamental Engineering Fluid Mechanics [M]. Beijing: Beijing Institute of Technology Press, 2012. (in Chinese)

[4] Wang Qidong, Wu Bofu, Zhao Liuqi. Integrated Control of Automobile Active Suspension and Electrical Power Steering Systems Based on Predictive Control [J]. Transactions of the Chinese Society for Agricultural Machinery, 2007, 38(1):1-5. (in Chinese)

[5] Li Shaoyuan, Li Ning. The Fuzzy Prediction Control of Complex System and Its Application [M]. Beijing: Science Press, 2003. (in Chinese)

[6] Yang Jiansen, Guo Konghui, et al. Vehicle integrated chassis control based on model predictive control [J]. Journal of Jilin University (Engineering and Technology Edition), 2011. 41(2): 1-5. (in Chinese)

[7] Wang Xiaofeng. Research on model prediction control methods based on can-bus networked control system [D]. Hang Zhou: Zhejiang University of Technology, 2009. (in Chinese)

[8] Zou Tao, Ding Baocang, Zhang Duan. MPC: An Introduction to Industrial Applications [M]. Beijing: Chemical Industry Press, 2010. (in Chinese) 